\title{
It’s All about Signals, Risk Management and How Important These Are?
}

\section{Garlapati $\mathbf{S}^{1 *}$ and Anireddy $\mathbf{K R}^{2}$}

${ }^{1} 3 R$ Biopharma, LLC 50 Dinsmore Ave, Framingham, MA, USA

${ }^{2}$ Vigilare Biopharma, Pvt. Ltd. Kukatpally, Hyderabad, India

\begin{abstract}
Pharmacovigilance is the study that amplifies safety of drugs with the fundamental objective of complete abstain on the ADRs and timely identification/detection of new adverse drug reactions. The fundamental principle of the pharmacovigilance is to figure out and valuate the benefit and risk profile of the pharmacological activity and to increase the rational usage of drugs. Adverse Drug Reactions (ADR) are a major cause of patient morbidity and mortality. Spontaneous reporting of ADRs is found to be crucial in maintaining patient safety.
\end{abstract}

Abbreviations: ADR: Adverse Drug Reactions; ICSR's: Individual Case Safety Reports

\section{Signal Detection}

Signal management is the important scientific component of the pharmacovigilance practice so has been placed at the prominent position which involves the set of activities performed to determine basing on the ICSR's and aggregated data from the studies and literature information available. It also involves set of activities to determine based on various data sources whether there are new/changed risks associated with active substances/medicinal products $[1,2]$.

The methods used for the signal detection should be applicable and relevant as inappropriate Large and complex statistical tools are not recommended for the smaller data. In case of review of ICSR's the complete methodology is precisely documented. A signal is considered urgent if it have an important public health impact or may significantly affect the benefit-risk profile of the medicinal product in treated patients $[3,4]$. A signal may be crucial or urgent if it have encounter a rap impact and it significantly affect the Benefit-risk profile of the medicine or a medical product treated in the group of subjects [5].

A typical signal detection program involves subsequently the following steps like;

1. Signal detection

2. Prioritization and

\section{Evaluation}

These are linked to the risk management and its activities. A safety signal has its specific life cycle and pharmacovigilance and signal detection are crucial activities of the drug or a medicinal product in its life cycle. Signals can be identified either manually or Automated signal detection methods [6].

\section{Conclusion}

Risk management is recommended to start at the early stages of clinical drug development process for all the drugs. A better understanding of the pharmacology and identification of the adverse drug reactions are often necessary to improve the strategies and aspects of pharmacovigilance. Usage of the methodology depends on type of adverse effects so as relying on any single method cannot be done. In the future a combination of quantitative and qualitative criteria may be incorporated in automated signal detection. New safety measures may be very uncertain and sensitive too.

\section{References}

1. Lateef MK Sameer EA, Huda MA, Abdel-Moneim MO, Ahmed SA (2014) Detection of Adverse Drug Reactions by medication antidote signals and comparison of their sensitivity with common methods of ADR detection. Saudi Pharmaceutical Journal.

2. European Medicines Agency (2012) Guidelines on good pharmacovigilance practices (GVP) Module IX-Signal management.

3. Pharmaceutical Information and Pharmacovigilance Association. GVP Module IX: Signal Management.

4. Meyboom RH, Egberts AC, Gribnau FW, Hekster YA (1999) Pharmacovigilance in perspective. Drug Saf 21: 429-447.

5. World Health Organization (2002) The importance of pharmacovigilancesafety monitoring of medicinal products. World Health Organization, Geneva, Switzerland.

6. CIOMS Working Group VIII (2010) Practical aspects of signal detection in pharmacovigilance. Council for International Organizations of Medical Sciences (CIOMS), Geneva
*Corresponding author: Garlapati S, 3R Biopharma, LLC 50 Dinsmore Ave Framingham, MA, USA, Tel: 1 -914-486-1898; E-mail: suman1981@gmail.com

Received November 04, 2014; Accepted November 05, 2014; Published November 06, 2014

Citation: Garlapati S, Anireddy KR (2014) It's All about Signals, Risk Management and How Important These Are? Adv Pharmacoepidemiol Drug Saf 3: e127. doi:10.4172/2167-1052.1000e127

Copyright: () 2014 Garlapati S, et al. This is an open-access article distributed under the terms of the Creative Commons Attribution License, which permits unrestricted use, distribution, and reproduction in any medium, provided the original author and source are credited. 